\title{
Skin layer recovery of free-surface wakes: Relationship to surface renewal and dependence on heat flux and background turbulence
}

\author{
C. J. Zappa and A. T. Jessup \\ Applied Physics Laboratory, College of Ocean and Fishery Sciences, University of Washington, Seattle \\ Department of Civil Engineering, University of Washington, Seattle
}

Harry Yeh

Department of Civil Engineering, University of Washington, Seattle

\begin{abstract}
The thermal signatures of free-surface wakes observed in the open ocean show that the recovery of the cool skin layer is related to the degree of surface mixing and to ambient environmental conditions. Wakes produced by two surface-piercing cables of $O\left(10^{-2} \mathrm{~m}\right)$ in diameter are analyzed using infrared imagery. Under low-wind-speed conditions when the swell and surface current were aligned, the wakes exhibited distinctive patchlike features of $O(1 \mathrm{~m})$ in diameter that were generated by the passage of individual waves. The time $t_{*}$ required by the skin layer to recover from these disturbances is compared to the surface-renewal timescale $\tau$ used in heat and gas flux models. At low wind speeds, $t_{*}$ is comparable to $\tau$, but at moderate wind speeds the agreement is poor. The spatial and temporal variations in the skin temperature of these wakes are related to a wave Reynolds number used to characterize the strength of the disturbance due to the waves. The recovery process is characterized in terms of the restoring internal energy flux $J_{r}$ which is proportional to both the initial thickness and the thermal recovery rate of the skin layer and was found to be directly related to the strength of the surface disruption. Comparison of the wake results with laboratory and other field measurements of breaking waves implies that $J_{r}$ is also a strong function of the net heat flux and background turbulence, which relate directly to the existing environmental conditions such as wind stress and sea state. Our results demonstrate that $J_{r}$ may vary by several orders of magnitude, depending on the environmental conditions.
\end{abstract}

\section{Introduction}

The temperature difference across the thermal boundary layer at the ocean surface is recognized as being relevant to satellitebased measurements of sea surface temperature [Schlüssel et al., 1990; Harris and Saunders, 1996] and to the accurate estimation of air-sea fluxes of gas and heat [Robertson and Watson, 1992; Van Scoy et al., 1995; Fairall et al., 1996]. Because the net heat flux through this thermal "skin layer" is normally from the ocean to the atmosphere, observations show that the "skin temperature" is typically $0.1^{\circ}$ to $0.5^{\circ} \mathrm{C}$ less than the bulk temperature immediately below [Katsaros, 1980; Robinson et al., 1984]. An infrared radiometer is ideally suited for measuring the skin temperature since the optical depth of $O\left(10^{-5} \mathrm{~m}\right)$ is much less than the skin layer thickness of $O\left(10^{-3} \mathrm{~m}\right)[$ McAlister and McLeish, 1969; Wu 1971; Hill, 1972]. Convincing evidence for the existence of the skin layer of the ocean was first provided by Ewing and McAlister [1960], who made infrared skin temperature measurements of the thermal recovery of surface disruptions produced by a pump and by breaking waves. Hasse [1990] proposed that the thermal recovery time of the skin layer $t_{*}$ may be used to estimate the

Copyright 1998 by the American Geophysical Union.

Paper number $98 \mathrm{JC} 01942$.

0148-0227/98/98JC-01942\$09.00 surface-renewal timescale $\tau$, which is the basis for surfacerenewal bulk-flux models. His estimate of $\tau \approx 10 \mathrm{~s}$, derived from typical ocean values of net heat flux and bulk-skin temperature difference, is comparable to the skin layer recovery times measured by Ewing and McAlister [1960] and by Katsaros [1977], as well as to the air-sea exchange timescales inferred by Jähne et al. [1989]. However, Ewing and McAlister [1960, p. 1374] also reported a range of skin layer recovery times and that "a remarkable finding was that less intense disturbance of the water failed to produce measurable effects." This early insight that the level of turbulence that disrupts the skin layer plays a significant role in the recovery process is supported by the recent results of Jessup et al. [1997a], who reported open-ocean $t_{*}$ values of $O\left(10^{-1} \mathrm{~s}\right)$ and furthermore found that the skin layer recovery rate in the wakes of breaking waves was correlated with a measure of the energy dissipation rate due to breaking.

Classical surface-renewal theory used in models for the flux of gas [Danckwerts, 1951] and heat [Liu et al., 1979] is based on the continuous random renewal of the surface by turbulent eddies from below. The idealized renewal process is one in which disruption is complete and instantaneous and recovery begins immediately. The average age of fluid elements occupying the surface is taken as the characteristic timescale for surface renewal $\tau$, which also is interpreted as the mean time between successive surface-renewal events. In reality, not all renewal eddies produce fluid-parcel overturning at the surface as illustrated in Figure 1a 
(a)
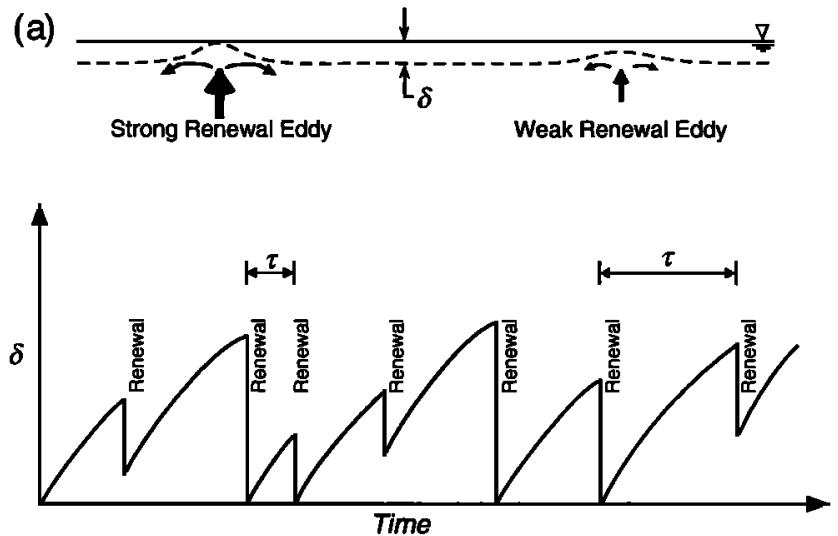

(b)

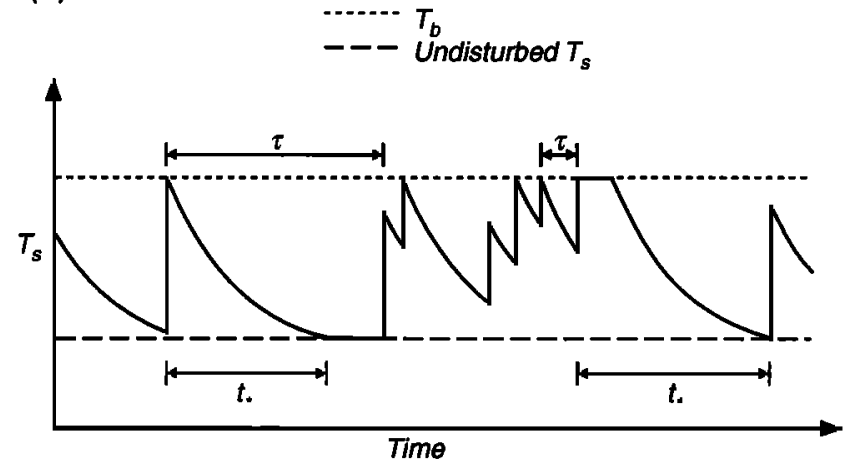

Figure 1. (a) (top) Conceptual sketch of the random-eddy penetration process [Harriott, 1962] occurring on the water side of the air-sea interface and (bottom) a time series of the boundary layer thickness $\delta$ at a single location (adapted from Gulliver [1991]). Turbulent eddies intermittently penetrate the boundary layer, forcing $\delta$ toward zero and allowing the underlying water to mix to the surface. (b) Idealized time series of sea surface temperature $T_{s}$ for a cool skin layer at a single location, illustrating the distinct difference between the skin layer recovery time $t_{*}$ and the surface-renewal timescale $\tau$. Skin layer recovery is the response to a disruptive event. As the turbulence decays, $T_{s}$ returns to its original value after a time $t_{*}$. In contrast, $\tau$ is the time between renewal events.

and as described in random-eddy penetration models [Harriott, 1962]. Accordingly, the thickness of the skin layer $\delta$ varies over space and time as eddies intermittently penetrate the layer.

In contrast to the surface-renewal timescale, the skin layer recovery time $t_{*}$ characterizes the diffusive process following disruption of the surface by a renewal eddy or other turbulent event. For a cool skin layer, Figure 1b illustrates how warmer bulk water mixes to the surface, so that immediately after a disruption the skin temperature $T_{s}$ is approximately equal to the bulk temperature $T_{b}$. As the turbulence gradually decays, $T_{s}$ returns to its original value after a time $t_{*}$. At times, multiple renewal events successively disrupt the skin layer before the initial disturbance has had a chance to recover fully. Furthermore, a sufficiently energetic and enduring disruption may delay the onset of recovery for a finite time. As the strength of the disturbance increases, the resulting increase in turbulence will force a longer $t_{*}$. While both the skin layer recovery time and surface-renewal timescale are undoubtedly affected by turbulence, Figure $1 \mathrm{~b}$ emphasizes that these two quantities may be distinctly different.

For shear-driven turbulence, $\delta$ can be related to the friction velocity in the water $u_{*}$ through dimensional analysis by

$$
\delta \propto \frac{v}{u_{*}},
$$

where $v$ is the kinematic viscosity. Saunders [1967a] asserted that the average bulk-skin temperature difference, $\Delta T=T_{b}-T_{s}$, can be described by

$$
\Delta T \propto-\frac{Q_{\text {net }} \delta}{k},
$$

where $k$ is the thermal conductivity and $Q_{\text {net }}$ is the net heat flux across the skin layer (positive into the ocean). For a given $Q_{\text {net }} \delta$ defines $\Delta T$ and is controlled by the near-surface turbulence intensity in the water. This interpretation of the dependence of $\Delta T$ on $\delta$ is consistent with the idea that the recovery of the skin layer is directly related to the decay of turbulence as observed by Ewing and McAlister [1960] and shown by Jessup et al. [1997a]. However, we expect that the skin layer recovery process will depend not only on the strength of the disruption but also on the net heat flux and the intensity of the background turbulence, as (1) and (2) imply.

Wick et al. [1996] have shown the similarity among theoretical models for $\Delta T$ when $\tau$ and $\delta$ are represented by the Kolmogorov time and length microscales as derived from the turbulent dissipation rate. Since $\tau$, as well as $\delta$, is not constant as the nature of turbulence changes, Soloviev and Schlüssel [1994] proposed a variable timescale for the surface-renewal process to account for the difference between turbulence generated in the freeconvective [Foster, 1971] and shear-driven [Csanady, 1990] regimes. Within a given regime, energetic turbulent events such as breaking waves may enhance the surface-renewal rate within the wake of a skin layer disruption.

Here we present nighttime observations and analysis of the infrared signatures of free-surface wakes generated by surfacepiercing cables deployed from R/P Flip under environmental conditions that varied from free to moderately forced convection. We demonstrate that the agreement between $\tau$ and $t_{*}$ depends on the environmental conditions. The skin layer recovery process is characterized in terms of the restoring internal energy flux $J_{r}$, which is directly proportional to both the initial thickness and the rate of thermal recovery of the skin layer. The results show that the skin layer recovery process is directly related to the strength of surface disruption and is dependent on the net heat flux, wind stress, and sea state.

\section{Field Experimental Procedure}

In January 1992, infrared images of the sea surface were collected 400 nautical miles $(740 \mathrm{~km})$ off the coast of San Diego aboard R/P Flip. Figure 2 shows the location of the primary instrumentation used in this study. We used an Agema model 880 $L W$ infrared imager operating in the spectral range of 8-12 $\mu \mathrm{m}$, which provided an integrated sea surface temperature over an effective depth of $10^{-5} \mathrm{~m}$. The noise-equivalent temperature difference of $0.05^{\circ} \mathrm{C}$ for the imager allowed the detection of minute variations in temperature for use as a flow visualization tool. The images were digitized at a frame rate of $6.25 \mathrm{~Hz}$ and have an im- 

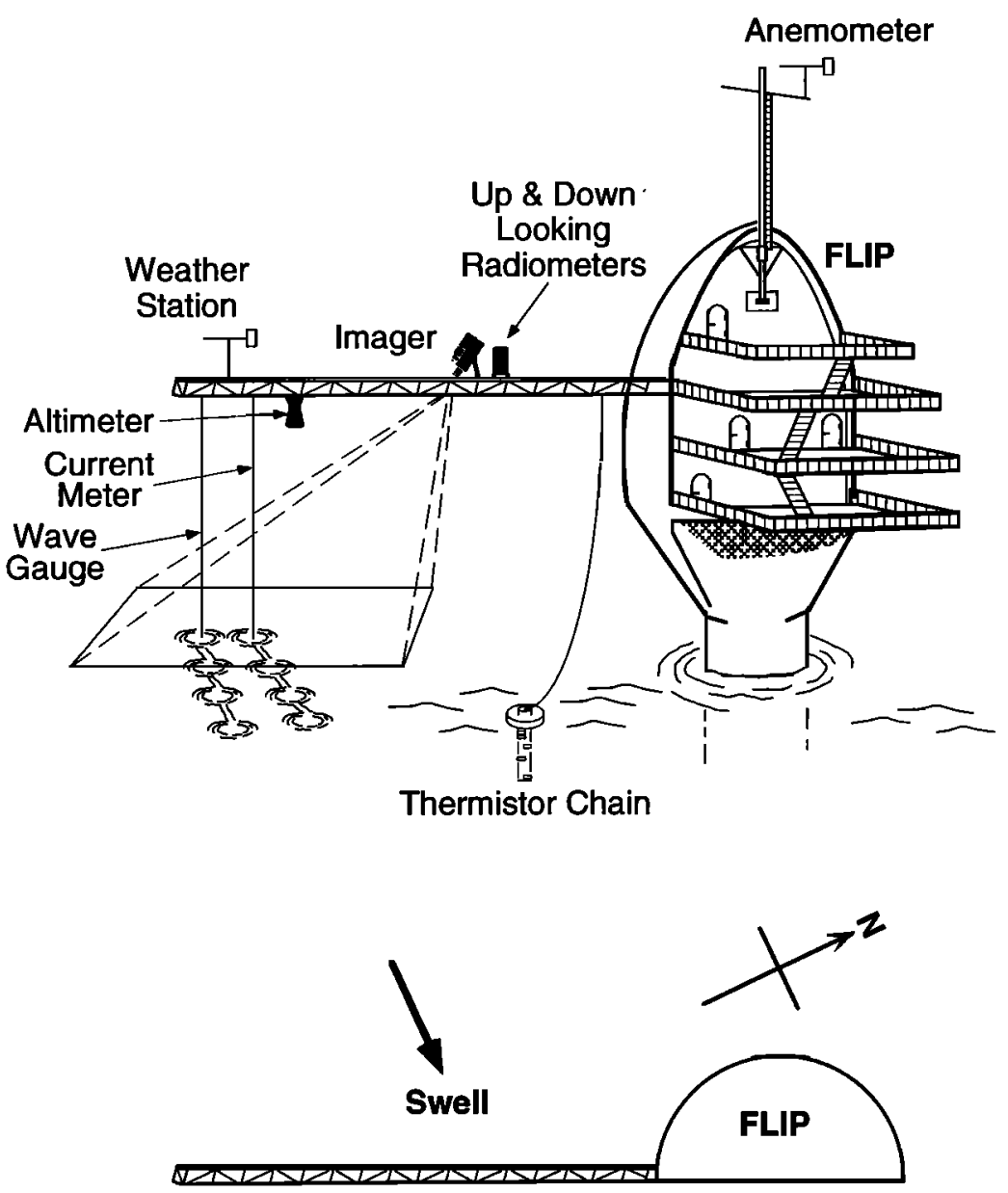

Figure 2. (top) Primary instrument deployment on the port boom aboard R/P Flip. (bottom) Plan view showing the orientation of R/P Flip with respect to the swell. The surface-piercing cables at the end of the boom generate two wakes with an infrared signature such as those shown in Plate 1 . The imager was oriented parallel to the instrument boom such that it viewed the surface directly under the boom. The swell approached from the west, and R/P Flip was oriented so as to produce the "ordered" wake structure.

age size of $140 \times 140$ pixels. We used a wide-angle lens to provide a $40^{\circ}$ field of view which significantly distorted the image, necessitating a correction scheme for quantitative analysis [Zappa, 1994]. At $10 \mathrm{~m}$ above the ocean surface and an incidence angle of $20^{\circ}$, the imager viewed an area roughly $7.7 \mathrm{~m}$ wide at the base, $10.0 \mathrm{~m}$ wide at the top, and $9.3 \mathrm{~m}$ long.

In conjunction with the infrared imager, we measured the skin temperature with a Heimann model KT-19 infrared radiation thermometer to an absolute accuracy better than $\pm 0.1^{\circ} \mathrm{C}[$ Zappa, 1994]. A wave-following thermistor chain of five Sea-Bird model SBE-3 oceanographic thermometers provided a vertical profile of the bulk water temperature in the top $5 \mathrm{~m}$. Combining the sea surface and bulk temperature measurements, we calculated $\Delta T$ using $T_{s}$ corrected for sky reflection (see Appendix A) and $T_{b}$ at a depth of $0.1 \mathrm{~m}$. Propeller and vane anemometers at heights of 10 and $25 \mathrm{~m}$ monitored the wind speed and direction. An aspirated slowresponse thermistor measured the air temperature, and a shielded Rotronics probe monitored the relative humidity. An Eppley model PSP pyranometer measured the solar irradiance, while an Eppley model PIR pyrgeometer measured the irradiant longwave radiation from the sky. Details of the calculation of net heat flux are described by Zappa [1994] and follow standard bulk formulations. An InterOcean Systems model S4 current meter measured the speed and direction of the ocean currents at a depth of approximately $5 \mathrm{~m}$. Finally, a wire wave gauge (Delavan model C/420-0 liquid level meter) and a scatterometer [Keller and Plant, 1990] used as an altimeter provided redundant measurements of sea surface displacement. During the experiment, the $1-\mathrm{cm}$ diameter wire wave gauge was modified by attaching 3-cm diameter PVC tubing cut into $3-\mathrm{cm}$-long sections spaced at $1-\mathrm{m}$ intervals. A $0.25-\mathrm{cm}$ diameter grounding cable was then attached to these PVC sections, separating the grounding cable from the wave gauge cable and increasing the effective diameter of the cable.

\section{Observations and Results of Free-Surface Wakes}

Figure 2 shows the two surface-piercing instrument cables that were deployed from the port boom of the freely drifting R/P Flip. The infrared signature of the disruption of the skin layer by the cables provided a means to detect and characterize the freesurface wakes that were continuously generated. The disturbance produced by the relative flow past the cables ruptured the cool skin layer and mixed the warmer bulk water from below to the surface. The appearance of the wake signatures varied with envi- 
ronmental conditions and was categorized as "ordered," "wispy," and "convective" (see Table 1). The pattern observed was directly related to the wind speed and the nature of the oscillatory flow past the cables generated by the swell in combination with the relative drift current.

Plate 1 is an infrared image of the ordered wakes, showing periodic, patchlike features within which the surface temperature was up to $0.4^{\circ} \mathrm{C}$ greater than the undisturbed skin temperature. This distinctive pattern occurred under low wind conditions when the waves and current were aligned. The conditions existing during the ordered wakes are shown schematically in Figure 2, where R/P Flip is drifting at $0.15 \mathrm{~m} \mathrm{~s}^{-1}$ at a bearing of $306^{\circ}$ with an orientation of $280^{\circ}$ (the keel points roughly into the wind). From recordings of the infrared images, we observed that the relative surface drift past R/P Flip was aligned with the swell which approached from the west. The large, turbulent patches coupled with the long, slender wakes indicate a periodic flow. The interaction of the swell waves with the current produced large variations in the relative flow past the cables, resulting in this distinctive periodic wake structure.

As the crest of a wave propagated past the relatively stationary cable, it induced a large surface motion that produced a long, slender wake. As a wave trough passed the cable, the current and orbital velocities counteracted each other, resulting in a lull in relative flow. During this period the cable continuously mixed the area within the recently disturbed slender wake, leading to the formation of large, patchlike features. Upon passage of yet another wave crest, the turbulent patch was accelerated away from the cable, increasing the level of disruption within the patch and forming another long, slender wake. The large circular turbulent patches were of $O(1 \mathrm{~m})$ in diameter, and the long, slender wakes were of $O\left(10^{-1} \mathrm{~m}\right)$ in width. Thus the wake features were roughly 100 times the $0.01-\mathrm{m}$ diameter $D$ of the cable.

Tracking the evolution of a single patch throughout its lifetime illustrates the difference in the growth and decay cycles of the temperature distribution and the wake width. Plate 2 shows image time series of a single patch formed by the passage of an individual wave with a period $T_{o}$ of $6.4 \mathrm{~s}$ and wave height $H_{o}$ of $1.0 \mathrm{~m}$. The period $T_{o}$ of individual waves was found by measuring the time between zero crossings on the upslope of the surface displacement time series. The wave height $H_{o}$ was taken as the difference between the maximum and minimum surface displacement within $T_{o}$. The first image, at time $t=0.0 \mathrm{~s}$, shows the uniform and undisturbed cool skin layer just prior to the initial disturbance. At $t=5.9 \mathrm{~s}$ the temperature within the patch reached a maximum, denoted by the white area within the images. At $t=$ $8.2 \mathrm{~s}$ the image shows the formation of a narrow wake, indicating that the patch was advected away from the cable at this time. The patch continued to grow in size, even though the cable no longer interacted within it, reaching maximum width at $t=13.1 \mathrm{~s}$ (roughly twice $T_{o}$ ). After this time the patch slowly decayed in size until it eventually drifted out of the field of view after approximately $41 \mathrm{~s}$.

Figure 3 shows cross-sectional profiles of the temperature of the patch in Plate 2 at different stages of development. The patch grew in size and increased in temperature from its initial stages until the temperature within the patch reached a maximum. That the patch continued to grow in size even though the temperature within the patch began to diminish implies the thermal recovery of the skin layer began to take place even as the turbulence continued to diffuse laterally. The temperature profiles across the patch suggest a Gaussian temperature distribution. The mean of the distribution represents the center of the patch, and 2 standard deviations provide a measure of the wake width. We defined the characteristic patch dimension, $b$, as the average of orthogonal wake widths. Also we defined the temperature difference across the patch $\Delta T_{w}$ as the difference between the maximum temperature $T_{\max }$ within the turbulent patch and the undisturbed skin temperature $T_{\text {amb }}$.

As the wakes formed continuously with the periodicity of the

Table 1. Environmental Conditions for the "Ordered" and "Wispy" Wakes Produced by the Two Cables and the "Convective" Patchiness

\begin{tabular}{|c|c|c|c|}
\hline & Ordered & Wispy & Convective \\
\hline Date & Jan. 26, 1992 & Jan. 28, 1992 & Jan. 26,1992 \\
\hline Time, LT & $1931-2040$ & $2235-2334$ & $2105-2205$ \\
\hline Wind speed, $U_{10}, \mathrm{~m} \mathrm{~s}^{-1}$ & $2.1 \pm 0.2$ & $7.3 \pm 0.4$ & $0.7 \pm 0.3$ \\
\hline Wind direction, deg & $284 \pm 9$ & $34 \pm 2$ & $\begin{array}{l}287 \pm 19(40 \mathrm{~min} .) \\
126 \pm 35(20 \mathrm{~min} .)\end{array}$ \\
\hline Keel heading, deg & $270 \pm 8$ & $18 \pm 4$ & $300 \pm 22$ \\
\hline Wave direction, deg & 270 & 270 & 280 \\
\hline Air temperature $T_{a},{ }^{\circ} \mathrm{C}$ & $14.65 \pm 0.05$ & $14.6 \pm 0.0$ & $14.5 \pm 0.1$ \\
\hline Relative humidity $R H, \%$ & $88.9 \pm 0.6$ & $89.2 \pm 0.6$ & $88.5 \pm 0.7$ \\
\hline $\begin{array}{l}\text { Bulk water temperature } T_{b} \\
\text { (uniform with depth), }{ }^{\circ} \mathrm{C}\end{array}$ & $14.34 \pm 0.01$ & $14.70 \pm 0.05$ & $1429 \pm 0.02$ \\
\hline Sea surface temperature $T_{s},{ }^{\circ} \mathrm{C}$ & $13.75 \pm 0.03$ & $14.51 \pm 0.04$ & $13.72 \pm 0.07$ \\
\hline Net heat flux $Q_{\text {net }}, \mathrm{W} \mathrm{m}^{-2}$ & $-94.0 \pm 2.9$ & $-128.3 \pm 3.6$ & $-884 \pm 4.5$ \\
\hline Friction velocity $u_{*}, \mathrm{~m} \mathrm{~s}^{-1}$ & $0.0022 \pm 0.0002$ & $0.0094 \pm 0.0006$ & $0.0007 \pm 0.0004$ \\
\hline $\begin{array}{l}\text { Surface Richardson number } \\
R f_{o}\end{array}$ & $-3.25 \times 10^{-3}$ & $-1.3 \times 10^{-5}$ & -1.4 \\
\hline
\end{tabular}



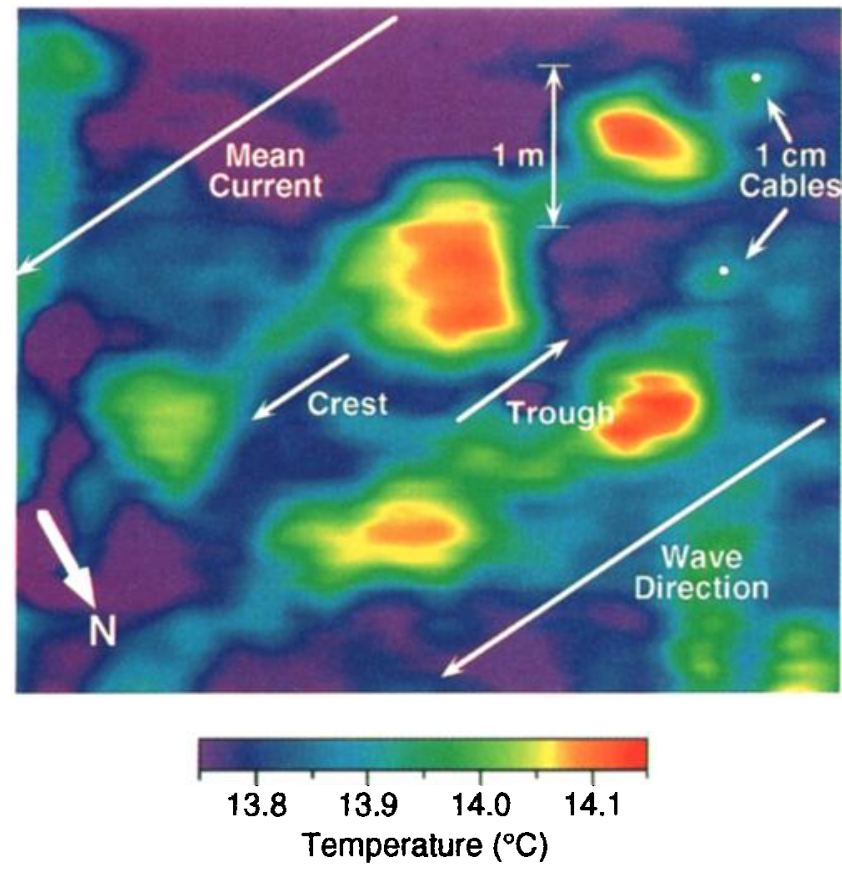

Plate 1. Infrared signature of ordered wakes emanating from two surface-piercing cables generated under low wind conditions by swell waves propagating in the direction of the mean current. The slender wakes were produced when the horizontal orbital velocity added to the mean current as the crest of the wave passed the cable. The large patches were produced during periods of low relative velocity when the trough passed. The cable diameter is of $O\left(10^{-2} \mathrm{~m}\right)$, and the patches are of $O(1 \mathrm{~m})$ in diameter (image size is $5 \mathrm{~m} \times 5 \mathrm{~m}$ ). The wind speed was $2 \mathrm{~m} \mathrm{~s}^{-1}$. The significant wave height was $3.7 \mathrm{~m}$.

wave field, we observed that longer and more energetic swell waves produced a longer lasting and stronger disturbance, resulting in larger and more persistent patches. Figure 4 shows three examples of time series of $b$ and $\Delta T_{w}$ that illustrate the effects of waves of different heights and periods. We characterized the disturbance caused by an individual wave by a wave Reynolds number, defined as

$$
R e_{w}=\frac{u_{o}}{v k_{o}}=\frac{a_{o} \omega_{o}}{v k_{o}}
$$

where $u_{o}=a_{o} \omega_{o}$ is the orbital velocity according to linear wave theory, $a_{o}=H_{o} / 2$ is the wave amplitude, $\omega_{o}=2 \pi / T_{o}$ is the wave frequency, and $k_{o}$ is the wavenumber based on the dispersion relation for linear waves.

Figure 4 shows the dependence $b$ and $\Delta T_{w}$ on $R e_{w}$ for three examples over the range of $R e_{w}$ encountered. We analyzed 15 ordered patches, for which $b_{m}$, the maximum value of $b$, ranged between 0.5 and $1.7 \mathrm{~m}$ and the patch lifetime was between 35 and $80 \mathrm{~s}$. Figure $4 \mathrm{a}$ shows that the width, the time required to reach the maximum width, and the lifetime of the patches all increased with increasing $R e_{w}$. For all patches, $b_{m}$ occurred well after the patch was advected away from the cable. Figure $4 \mathrm{~b}$ shows that for all the patches analyzed, $\Delta T_{w}$ rapidly increased to its maximum and that its decay was approximately linear with time. Closer observation of the time series of the wake signatures showed that the maximum $\Delta T_{w}$ increased linearly from $0.4^{\circ}$ to $0.5^{\circ} \mathrm{C}$ with increasing $R e_{w}$, suggesting that the skin layer may not have been completely destroyed by weaker disturbances. None of the patches completely recovered before drifting out of the field of view. Extrapolating the complete evolution and decay cycle for the patch with the largest $\operatorname{Re}_{w}$ produced a lifetime of $120 \mathrm{~s}$.

Jessup et al. [1997a] recently demonstrated that the skin layer recovery rate scales with the energy dissipation rate in the wakes of breaking waves. Larger breaking waves dissipate more energy and produce more turbulence, resulting in slower skin layer recovery rates. For dimensional consistency their results were presented in terms of a "restoring heat flux" which they defined as the rate of heat transfer per unit area required to reestablish the skin layer. Here we reinterpret this parameter and identify it by the more physically descriptive designation of the restoring internal energy flux $J_{r}$. When the cool skin layer is disturbed and replaced by warmer water from below, heat must be lost within a layer of original thickness $\delta$ for the cool skin layer to recover. Therefore the restoring internal energy flux is defined as the rate of decrease in internal energy of a cool skin layer per unit area during the recovery process and is given by

$$
J_{r}=\left(\rho c_{p} \delta\right) R_{t},
$$

where $\rho$ is the density of water, $c_{p}$ is the specific heat of water, and $R_{t}$ is the recovery rate of the skin layer. Jessup et al. [1997a] showed that $J_{r}$ was correlated with measures of the energy dissipation rate per unit area due to large-scale breaking waves in the field and laboratory.

In general, the thermal and spatial signatures of the patches show that the skin layer disruptions produced by larger waves re-
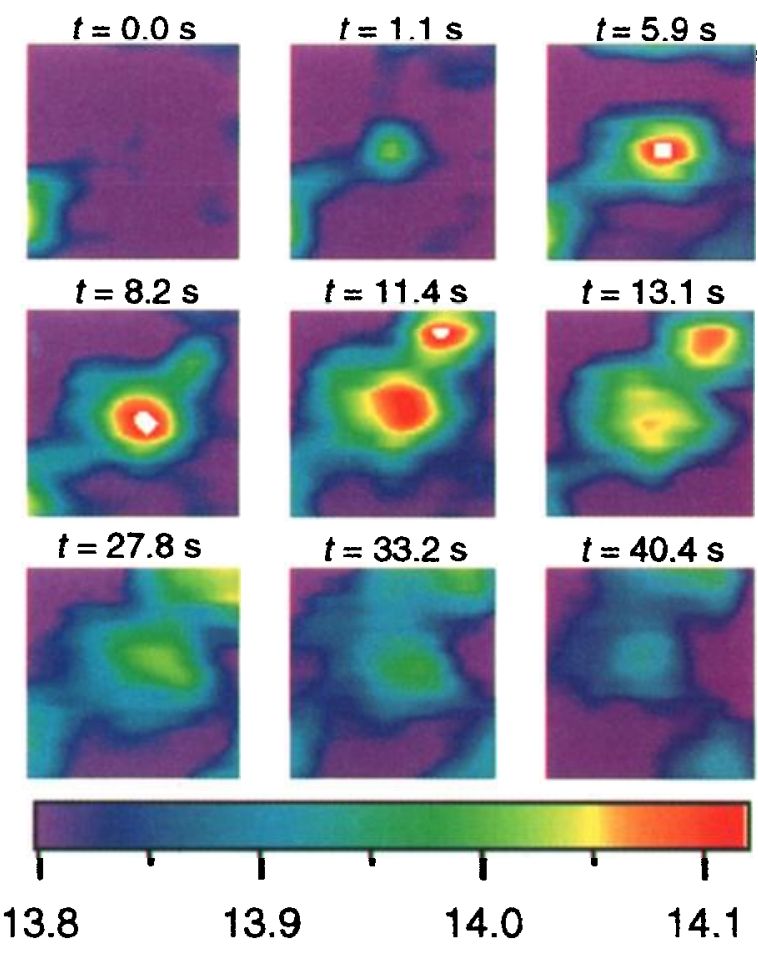

\section{Temperature $\left({ }^{\circ} \mathrm{C}\right)$}

Plate 2. Time series of infrared images (with irregular time step) following one particular turbulent patch. The image size is roughly $1.5 \mathrm{~m} \times 1.5 \mathrm{~m}$. After the temperature within the patch reaches a maximum $(t=5.9 \mathrm{~s})$, the patch continues to grow in size while the temperature begins to decrease. After reaching maximum spatial growth $(t=13.1 \mathrm{~s})$, the patch slowly decays in size as it propagates out of the image after roughly $41 \mathrm{~s}$. 


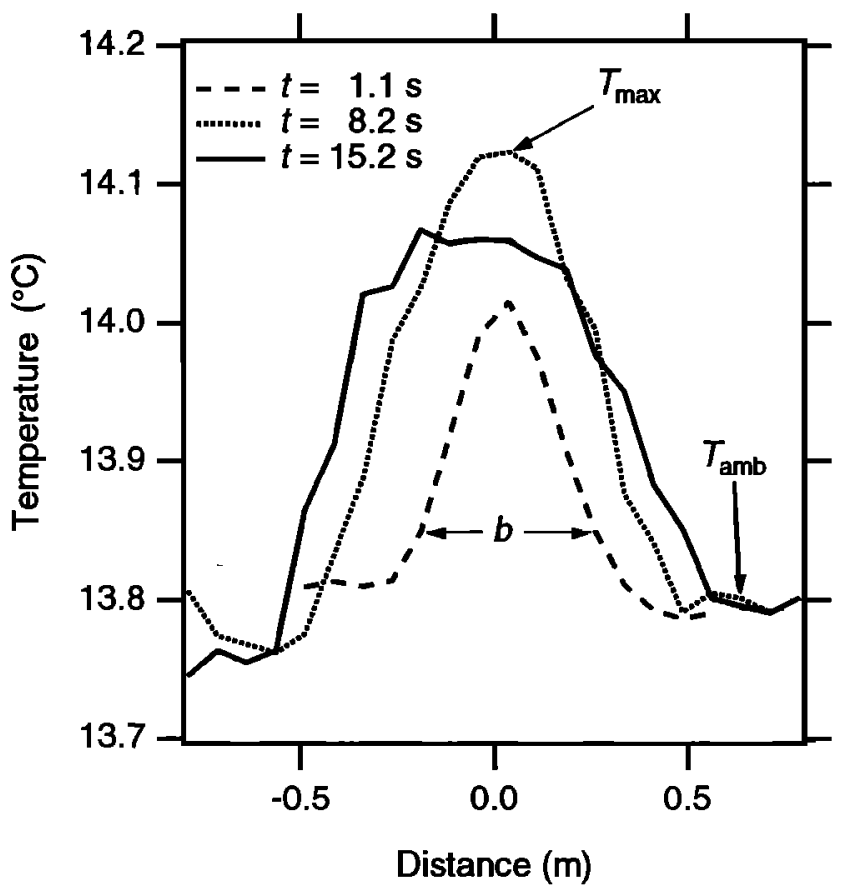

Figure 3. Cross-sectional profiles of the temperature of the wake in Plate 2 at different stages of development. The characteristic wake dimension $b$ defines the size of the patch, and the temperature difference across the patch is $\Delta T_{w}=T_{\max }-T_{\text {amb. }}$. Although the temperature within the patch begins to diminish after reaching $T_{\max }$, the patch continues to enlarge.

covered more slowly than those due to smaller waves. For each patch we computed a mean $R_{t}$ as the absolute value of the slope of a linear fit to the time series of $\Delta T_{w}$ starting at its maximum, as illustrated in Figure $4 b$. Since we did not directly measure the skin layer thickness, $\delta$ is determined from published values for the given wind conditions $[W u, 1971 ;$ Hill, 1972; Katsaros et al., 1977]. In Figure 5, $J_{r}$ for each patch, as computed from (4) with $\delta$ $=2 \mathrm{~mm}$, is plotted as a function of $R e_{w}$. The result shows that $J_{r}$ is correlated with $R e_{w}$, which characterizes the strength of the disruption, such that patches described by larger $R e_{w}$ recover more slowly. Figure 6 shows a similar plot for the patch width recovery rate $R_{b}$, which is calculated in a manner similar to $R_{l}$ and also decreases with increasing $R e_{w}$.

At higher wind speeds, between 5 and $10 \mathrm{~m} \mathrm{~s}^{-1}$, the disturbances from the cables remained visible but were very small and of short duration because of the rapid skin layer recovery rate due

Figure 4. (a) Time evolution of patch width $b$ for three wakes with different wave Reynolds numbers $R e_{w}$, each produced by an individual wave. Waves with larger $R e_{w}$ produce larger wakes that take longer to reach maximum size, resulting in longer skin layer recovery times. Skin layer disruptions lasting up to $60 \mathrm{~s}$ were measured, after which time they traveled out of the field of view. Extrapolating the trend for the largest $R e_{w}$ event observed gives a skin layer recovery time of approximately $120 \mathrm{~s}$. (b) Time series of maximum temperature difference between the patch and the undisturbed surface for the three patches in Figure 4a. Although each patch rapidly reaches a maximum $\Delta T_{w}$, the rate of thermal recovery of the skin layer is a function of $R e_{w}$. The slope of the line fitted from the maximum $\Delta T_{w}$ is taken as the skin layer recovery rate $R_{t}$ to the increased heat flux. Figure 7 shows a snapshot of these wispy wakes (see Table 1 for environmental conditions), which are more clearly evident in a recording of the imagery played at high speed. In the top left corner of Figure 7, the wakes appear as small bright features at the bottom of thin dark bands, which correspond to the wires. These wake features lasted for merely a few image frames or of $O\left(10^{-1} \mathrm{~s}\right)$, verifying the rapid recovery of the skin layer under these conditions. The occurrence of any wake signature at these higher wind speeds does not agree with the results of Konda et al. [1994], who concluded that the skin layer disappears at wind speeds above $5 \mathrm{~m} \mathrm{~s}^{-1}$ owing to intense surface mixing caused by the high shear stress. P. J. Minnett (personal communication, 1998) has also observed a $\Delta T$ near $0.2^{\circ} \mathrm{C}$ at wind speeds up to $15 \mathrm{~m} \mathrm{~s}^{-1}$ using an interferometer to measure $T_{s}$.

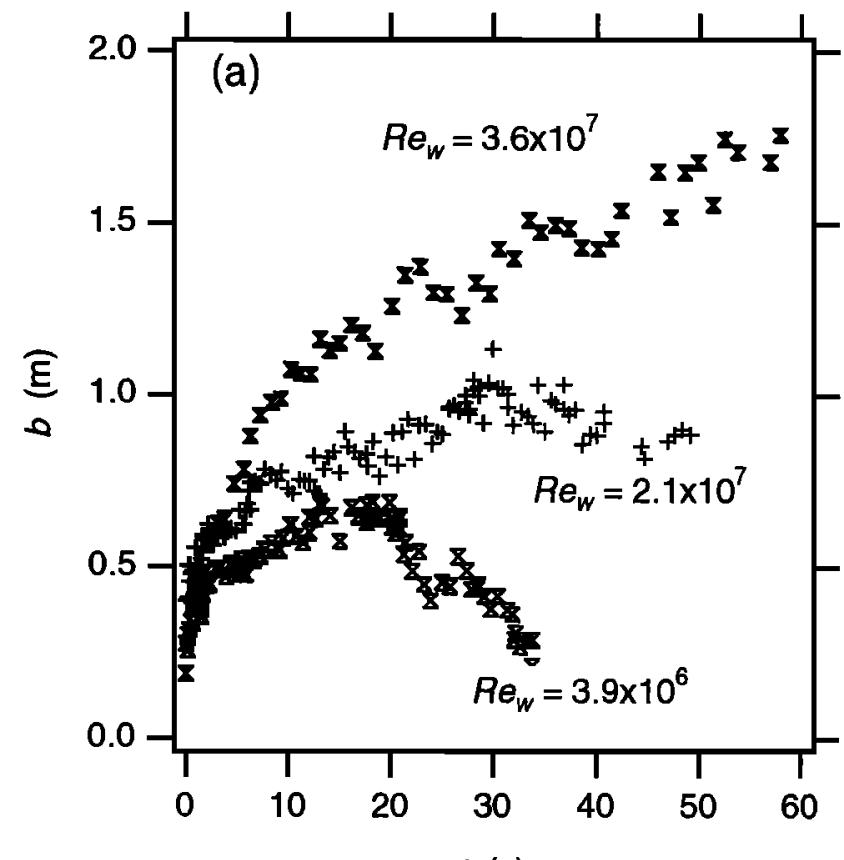

$t(\mathbf{s})$

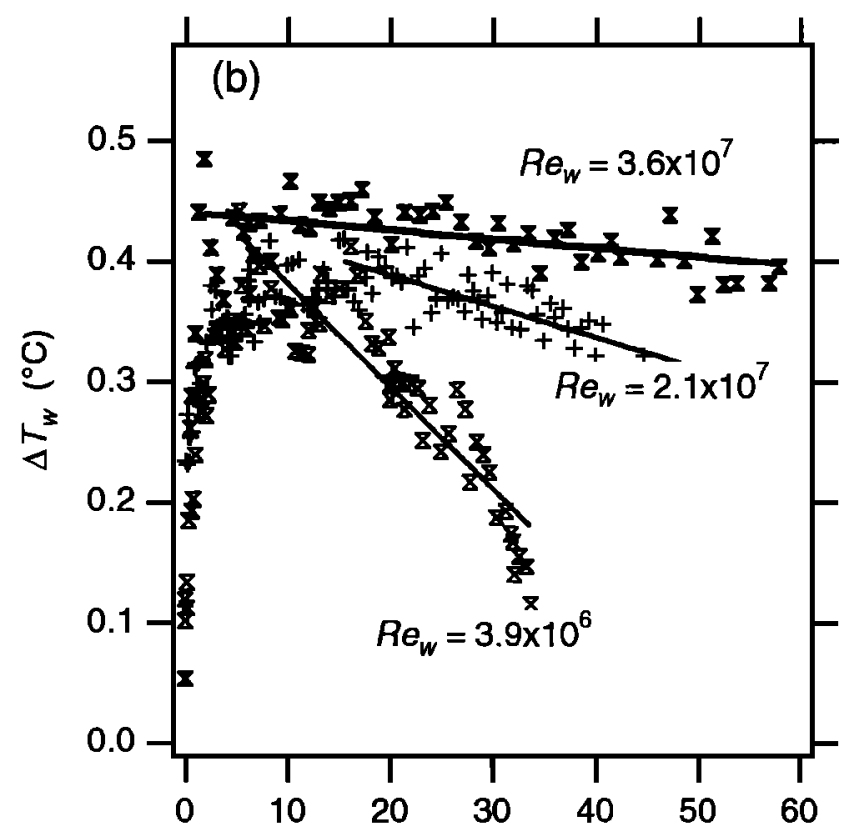

$t(\mathrm{~s})$ 


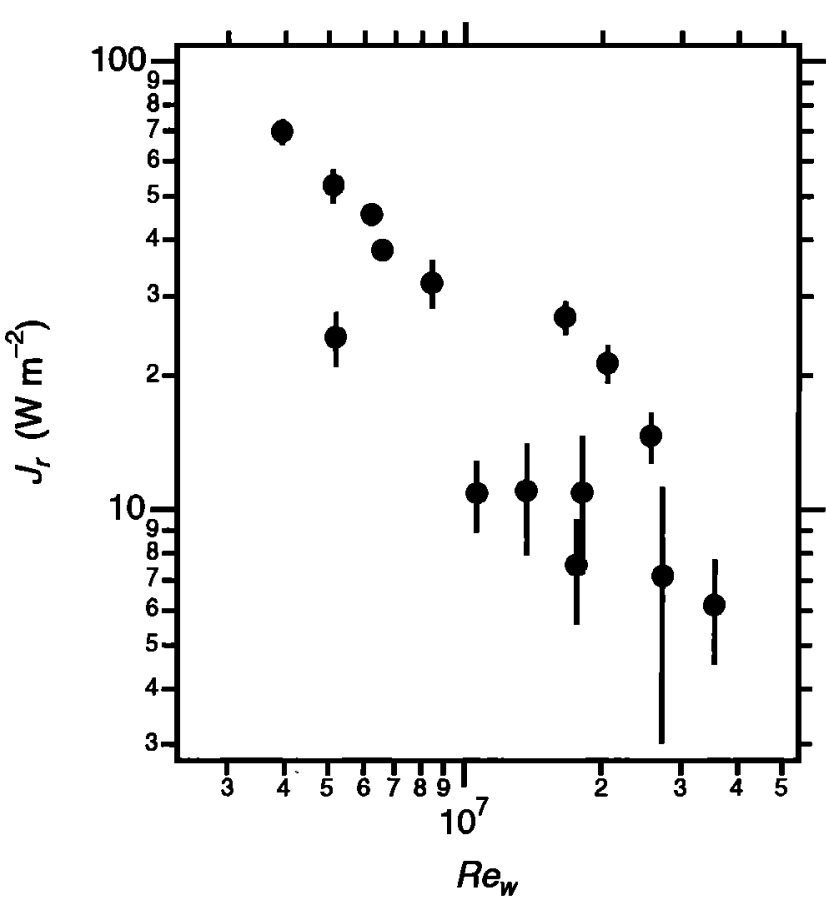

Figure 5. Restoring internal energy flux $J_{r}$ versus $R e_{w}$ for 15 individual patches. Since the net heat flux was constant, the result shows that the strength of the surface disruption determined $J_{r}$, such that patches described by a larger $R e_{w}$ recover more slowly. The error bars reflect the accuracy of the linear regression used to determine $R_{t}$

\section{Discussion}

\subsection{Comparison of Observed Skin Layer Recovery Times With Parameterized Surface-Renewal Timescales}

We have measured the infrared signatures of the ordered wakes under very low wind speeds $\left(-2.1 \mathrm{~m} \mathrm{~s}^{-1}\right)$ and of the wispy wakes under moderate wind speeds $\left(\sim 7.3 \mathrm{~m} \mathrm{~s}^{-1}\right)$. The net upward heat flux was roughly -94 and $-128 \mathrm{~W} \mathrm{~m}^{-2}$, respectively, resulting in a cool skin layer in both cases. Following disruption, the skin layer recovers to a thickness characterized by the level of turbulence in the water within the wake. As the turbulence level at the interface diminishes, the skin layer thickens and $\Delta T$ increases ever closer to its initial value. This may explain the increase in maximum $\Delta T_{w}$ from $0.4^{\circ}$ to $0.5^{\circ} \mathrm{C}$ as a function of increasing $R e_{w}$ for the ordered wakes. At low $R e_{w}$, weak turbulence within the wake may not completely destroy the cool skin and therefore $\Delta T_{w}$ may slightly underestimate $\Delta T$ (see Appendix B). Nevertheless, the level of turbulence generated in the wake of the cable was responsible for its slow recovery, implying that the timescale for skin layer recovery is directly related to the level of disruption.

Hasse [1990] proposed that the skin layer recovery time $t_{*}$ may be used to estimate the surface-renewal timescale $\tau$. Since our results vary over conditions ranging from low to moderate wind speed regimes, we evaluate the comparison using the parameterizations for the time scale of surface renewal proposed by Soloviev and Schlüssel [1994]. At low wind speeds $\left(U_{10}<\right.$ $5 \mathrm{~m} \mathrm{~s}^{-1}$ ) they suggested that the characteristic $\tau$ follows the injection model of Foster [1971]. According to Foster's model, inter- mittent convection provides the mechanism for surface renewal on a timescale of

$$
\tau_{c}=C_{c}\left(\frac{v \rho c_{p}}{\alpha g Q_{\mathrm{net}}}\right)^{1 / 2},
$$

where $C_{c}$ is a dimensionless constant, $g$ is the acceleration due to gravity, and $\alpha$ is the coefficient of thermal expansion of water. For moderate wind conditions $\left(U_{10}=5-10 \mathrm{~m} \mathrm{~s}^{-1}\right)$, Soloviev and Schlüssel [1994] suggest that the most intense surface renewal is caused by surface shear-stress variations associated with microscale wave breaking, as proposed by Csanady [1990]. According to Csanady's model, the timescale for surface renewal is

$$
\tau_{s}=C_{s} \frac{v}{u_{*}^{2}}
$$

where $C_{s}$ is a dimensionless constant. The constants $C_{C}$ and $C_{S}$ for our data were determined independently to be 15.4 and 554 , respectively [Wick and Jessup, 1998].

Soloviev and Schlüssel [1994] characterized the transition from free to forced convection within the skin layer with the surface Richardson number $R f_{o}$, defined by Kudryavtsev and Soloviev [1985] as

$$
R f_{o}=\frac{\alpha v g Q_{\mathrm{net}}}{\rho c_{p} u_{*}^{4}}
$$

This parameter describes the ratio of buoyancy to shear forcing, and the form in (7) is valid for our data since the calculated surface salt flux was a minor part of the surface buoyancy flux $(<5 \%)$. The critical value for the surface Richardson number is determined as $R f_{c r}=-\left(C_{c} / C_{s}\right)^{2}$ and for our data is $-7.7 \times 10^{-4}$. This value is of the same order as those determined by Soloviev and Schlüssel [1994] and Wick et al. [1996]. When $R f_{o}$ ap-

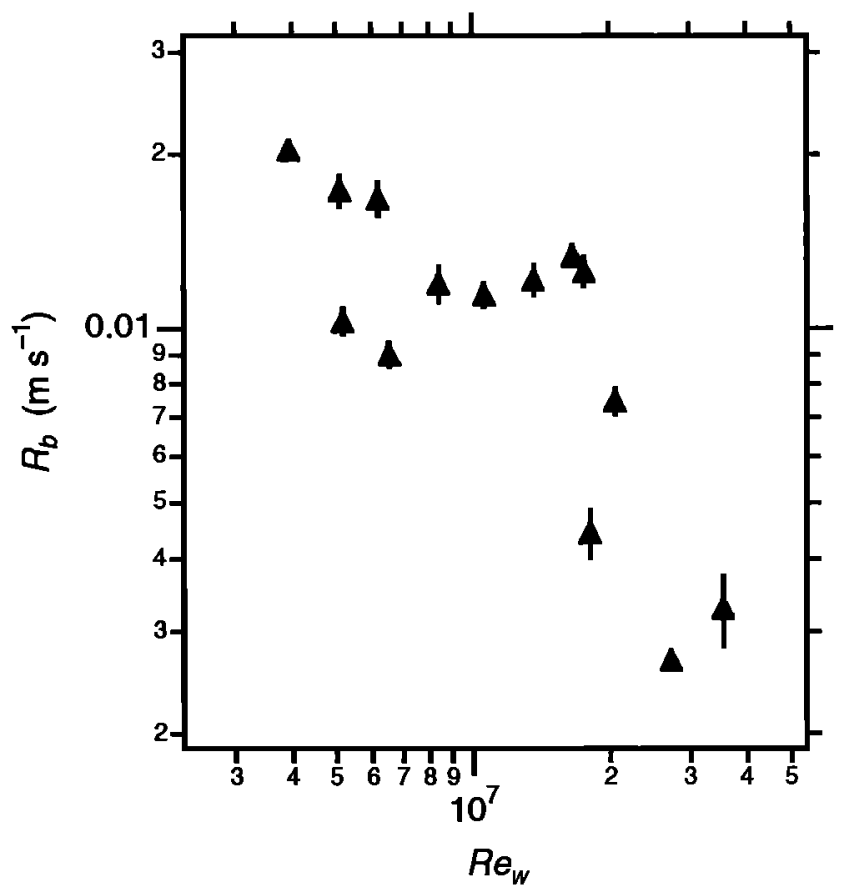

Figure 6. Patch width recovery rate $R_{b}$ for 14 individual patches versus $R e_{w}$. Similar to $J_{r}$ in Figure $5, R_{b}$ decreases with increasing $R e_{w}$. The error bars are analogous to those shown in Figure 5. 

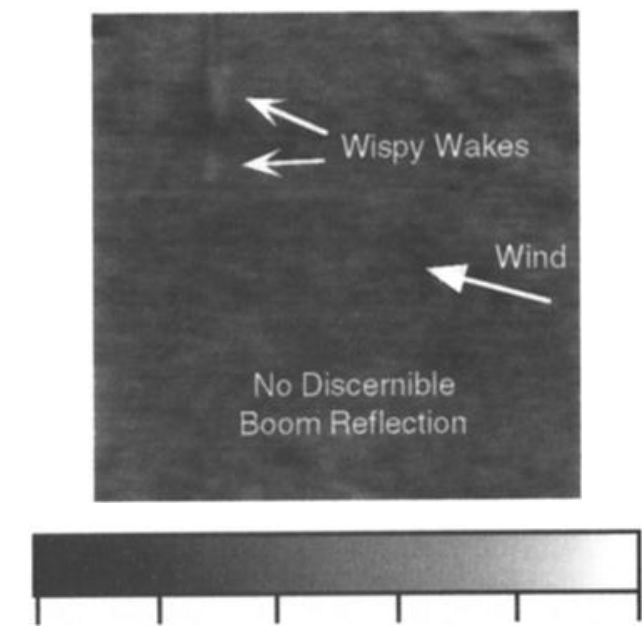

\section{$\begin{array}{llllll}14.3 & 14.4 & 14.5 & 14.6 & 14.7 & 14.8\end{array}$ Temperature $\left({ }^{\circ} \mathrm{C}\right)$}

Figure 7. Snapshot of "wispy" wakes emanating from the surface-piercing cables. The digitally enhanced, dark, vertical bands at top left are the cables, and the faint white features at the bottom of the cables are the wispy wakes, which last for merely a few image frames, or of $O\left(10^{-1} \mathrm{~s}\right)$. Note the lack of a discernible boom reflection (compare with Figure 8 ). The wind direction is from right to left.

proaches $R f_{c r}$, the production of turbulence by buoyancy is of the same order as the production of turbulence by shear stress; more negative values of $R f_{o}$ imply increasing importance of buoyancy forces.

For the ordered wakes, $R f_{o}$ was $-3.25 \times 10^{-3}$, indicating free convection conditions, and $\tau_{c}=67.6 \mathrm{~s}$. This value is comparable to the measured $t_{*}$ values ranging from 35 to $120 \mathrm{~s}$, suggesting that for this low-wind-speed regime, $t_{*}$ may be appropriate for
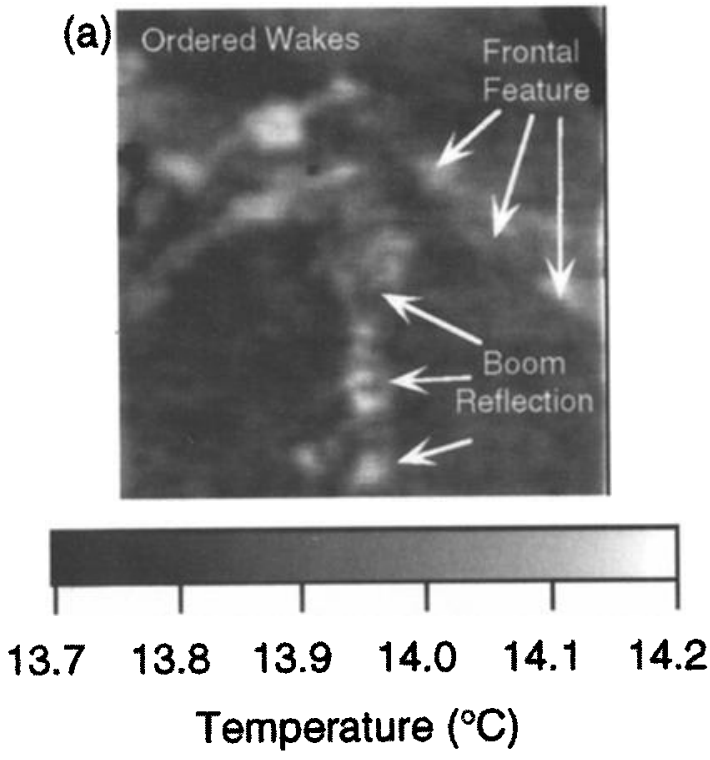

estimating $\tau$. Our resuits, however, show that $t_{*}$ is a function of the strength of the disturbance since the environmental conditions remained constant whereas $t_{*}$ varied with $R e_{w}$. For the wispy wakes, $R f_{o}$ was $-1.3 \times 10^{-5}$, indicating that shear played the dominant role in the near-surface processes, and $\tau_{s}=6.3 \mathrm{~s}$. This value is nearly an order of magnitude greater than the measured value of $t_{*}$ of $O\left(10^{-1} \mathrm{~s}\right)$. During this same time period, wakes produced by breaking waves had comparable recovery times [Jessup et al., 1997a]. These results suggest either that $t_{*}$ may not be an appropriate estimate for $\tau$ in this shear-driven regime or that $\tau_{s}$ is an inappropriate parameterization for $\tau$. Nonetheless, a comparison of the ordered wake results and the wispy wake results demonstrates that $t_{*}$ depends on the intensity of background turbulence and the net heat flux.

Infrared images of the sea surface reveal characteristic features that can be attributed to these specific turbulence regimes. The uniform background for the wispy wakes depicted in Figure 7 was produced by the well-mixed near-surface layer and is typical of our infrared observations made during shear-driven conditions. In contrast, the infrared images in Figure 8 illustrate the transition into the regime of intense buoyancy-produced turbulence near the air-water interface. Figure 8a shows the full image of the ordered wakes highlighted in Plate 1 . Unlike the uniformity exhibited during the wispy wake observations, frontal features, like the one in the right-hand side of Figure $8 \mathrm{a}$, continually propagated through the images (see Appendix A regarding the boom reflection that contaminates the bottom half of the image). These frontal features suggest the existence of near-surface, coherent structures as observed under very similar conditions by Soloviev [1990]. Although $R f_{o}$ at this time indicates free-convective conditions, the infrared image in Figure 8a does not exhibit any obvious convective features.

Subsequent to the ordered wake observations, $U_{10}$ dropped to less than $1 \mathrm{~m} \mathrm{~s}^{-1}$, resulting in an hourly averaged $R f_{o}$ value near -1.4. These conditions, as well as the small ocean currents $\left(<0.25 \mathrm{~m} \mathrm{~s}^{-1}\right.$, on average, for the experiment), indicate that buoyancy-driven processes dominated near the surface. In con- (b)
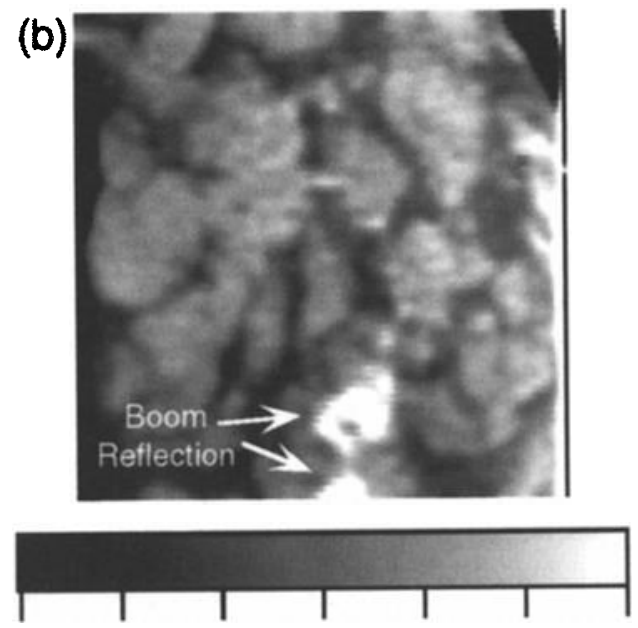

13.6
13.8

14.0

Temperature $\left({ }^{\circ} \mathrm{C}\right)$

Figure 8. Comparison of (a) ordered wakes and (b) "convective" patchiness. Images are identical in size (roughly $10 \mathrm{~m} \times 10 \mathrm{~m}$ ) and orientation (parallel to the boom) to Figure 7. The ordered wakes in Figure 8a also exist in Figure $8 \mathrm{~b}$ and are the same as those highlighted in Plate 1. Note the boom reflections in the bottom half of each image (see Appendix A). 
trast to the ordered wakes shown in Figure 8a, Figure $8 \mathrm{~b}$ shows a puffy patchiness that existed under these strongly free convective conditions. These convective features are comparable in size and $\Delta T_{w}$ to the ordered wake signatures in Figure 8a; note that the ordered wakes and the boom reflection of Figure 8a also appear in Figure $8 \mathrm{~b}$. The convective features persisted for several minutes while propagating through the images, suggesting that the turbulence generated within these patches was comparable to that in the ordered wakes. Figure 8 clearly shows the transition to intense buoyancy-driven turbulence that is strong enough to disrupt the skin layer. That similar features are not apparent in Figure 8a may be because the scale of convection within the skin layer is smaller than the spatial resolution of the imager.

The recovery times of $O\left(10^{2} \mathrm{~s}\right)$ that we observed at low wind speeds have not been previously reported in the literature and are in sharp contrast to the recovery times of $O\left(10^{-1} \mathrm{~s}\right)$ that we observed at moderate wind speeds. Under similar low-wind conditions, Ewing and McAlister [1960] reported recovery times of $5 \mathrm{~s}$ for a disturbance from an upwelling pump and $12 \mathrm{~s}$ for a breaking wave, suggesting that skin layer recovery depends on the turbulence generated at the interface. While other field investigators concur with Ewing and McAlister [1960] that the cool skin layer recovers within roughly 10 to $12 \mathrm{~s}$ [Katsaros, 1977; Schlüssel et al., 1990], we observed that the strength and type of the disruption, as well as the environmental conditions, are important variables in the skin layer recovery.

\subsection{Dependence of Skin Layer Recovery Rate on the Strength of Disturbance and Net Heat Flux}

Skin layer recovery times are difficult to determine precisely because the disturbances cannot always be tracked until complete recovery. Sometimes, successive events will disrupt the skin layer before the initial disturbance has had a chance to recover fully. Measuring the skin layer recovery rate $R_{t}$ alleviates these problems and provides additional information about the magnitude of $\Delta T$. As demonstrated in Figure 5, the results of our field study of ordered wakes suggest a direct relation between $J_{r}$, which is proportional to $R_{b}$, and the level of turbulence produced by the disruption. At low $R e_{w}$ the maximum $\Delta T_{w}$ and $b_{m}$ occurred almost simultaneously. Unable to sustain disruption, the low turbulent energy available within the wakes decayed quickly and the skin layer had a moderate $J_{r}$ of $70 \mathrm{~W} \mathrm{~m}^{-2}$ and a patch width recovery rate $R_{b}$ of $0.02 \mathrm{~m} \mathrm{~s}^{-1}$. As $R e_{w}$ increased, the $\Delta T_{w}$ maximum preceded $b_{m}$, leading to a low $J_{r}$ of $6 \mathrm{~W} \mathrm{~m}^{-2}$ and $R_{b}$ of $0.003 \mathrm{~m} \mathrm{~s}^{-1}$ because more turbulent energy was available to impede the skin layer's recovery. For a given net heat flux and skin layer thickness these results show that the strength of the disturbance deter$\operatorname{mined} J_{r}$.

Further support for the hypothesis that the thermal recovery rate depends on the strength of the disturbance is provided by a comparison of our results with the results of laboratory measurements by Jessup et al. [1997a]. For individual, mechanically generated breaking waves, they computed $R_{t}$ as the slope of the temperature deviation over 30 wave periods after breaking occurred; the air-water temperature difference $\Delta T_{a w}$ was constant at $-4.22 \pm 0.13^{\circ} \mathrm{C}$, and there was no wind $(\delta=3 \mathrm{~mm})$. Similar to the dependence of $J_{r}$ on $R e_{w}$ for the ordered wakes (see Figure 5), the correlation between $J_{r}$ and the energy dissipation rate per unit area $E$ for their laboratory breaking waves confirms that the restoring internal energy flux depends on the turbulence generated by the event. However, $J_{r}$ ranged between 60 and $140 \mathrm{~W} \mathrm{~m}^{-2}$, slightly greater than the magnitude found for the ordered wakes.
According to (2), not only should the restoring internal energy flux be a function of turbulence, but it also should depend on the net heat flux. The dependence of $R_{t}$ on the net heat flux for laboratory breaking waves was reported by Jessup et al. [1995]. We have analyzed additional data from their experiment and computed $R_{t}$ in the manner described by Jessup et al. [1997a]. Figure 9 shows $J_{r}$ versus $\Delta T_{a w}$ for all $E$ ranging from 2.03 to $2.88 \mathrm{~W} \mathrm{~m}^{-2}$ and no wind. Since the relative humidity remained nearly constant at $62.3 \pm 2.6 \%$ and there was no wind, $Q_{\text {net }}$ depended only on $\Delta T_{a w}$. Note that $J_{r}$ ranged from 20 to $200 \mathrm{~W} \mathrm{~m}^{-2}$ and encompassed the range of magnitudes from the constant net heat flux cases of both the ordered wakes and the laboratory breaking waves. More significantly, the correlation in Figure 9 shows higher restoring internal energy fluxes for more negative $\Delta T_{a w}$ or, alternatively, larger upward net heat flux. The result from Figure 9 suggests that $J_{r}$ is dependent more on $Q_{n e t}$ than on the strength of the disturbance. This $Q_{n e t}$ dependence may explain the difference in the mean magnitude of $J_{r}$ between the constant net heat flux cases of the ordered wakes and laboratory breaking waves.

\subsection{Dependence of $J_{r}$ on the Background Turbulence}

Results for low and no wind conditions have shown comparable moderate restoring internal energy fluxes ranging from 6 to $200 \mathrm{~W} \mathrm{~m}^{-2}$ for ordered, free-surface wakes and laboratory breaking waves. Our field results show that at a wind speed of $7.3 \mathrm{~m} \mathrm{~s}^{-1}$, the total recovery of the wispy wakes occurred 2 to 3 orders of magnitude sooner and was dominated by the accelerated net heat flux (roughly $-128 \mathrm{~W} \mathrm{~m}^{-2}$ ) and thinner skin layer $(\delta=$ $0.5 \mathrm{~mm}$ ) owing to the enhanced transfer of shear stress across the

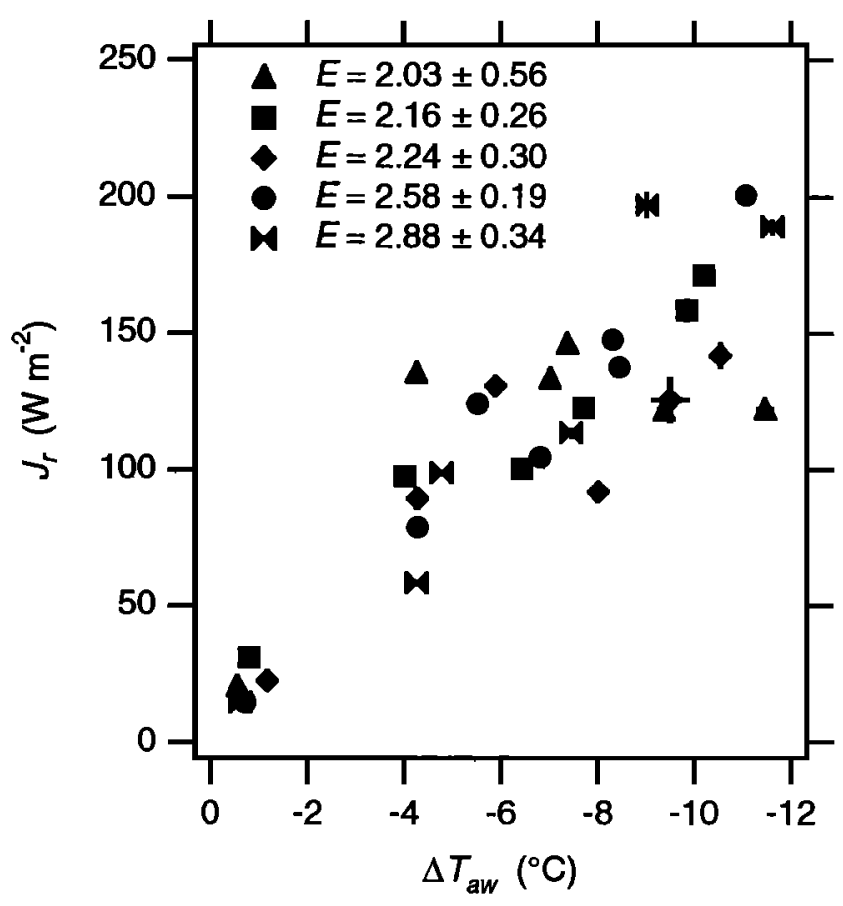

Figure 9. Restoring internal energy flux $J_{r}$ versus the air-water temperature difference $\Delta T_{\alpha w}$. The energy dissipation rate per unit area $E$ for breaking waves generated mechanically in the laboratory ranged from 2.03 to $2.88 \mathrm{~W} \mathrm{~m}^{-2}$, and there was no wind. The correlation shows higher $J_{r}$ for more negative $\Delta T_{a w}$ or, alternatively, larger upward net heat flux. Each point is an ensemble average of five repetitions, and the error bars represent 1 standard deviation. 
air-water interface. These two mechanisms prohibited the cable from interacting with its own wake as in the ordered wake case, thus producing less of a disturbance and resulting in $J_{r}$ of 200 $\mathrm{W} \mathrm{m} \mathrm{m}^{-2}$. The turbulent wakes of breaking waves, which occurred during the same time period as the wispy wakes, were much larger and more energetic disturbances and produced a $\Delta T_{w}$ comparable to $\Delta T$. The dependence of the restoring internal energy flux on a measure of the energy dissipation rate for these breaking waves in the field showed high $J_{r}$ values of roughly 200 to $1000 \mathrm{~W} \mathrm{~m}^{-2}$ [Jessup et al, 1997a], comparable to those for the wispy wakes.

The difference between the magnitudes of $J_{r}$ in the low and moderate wind speed regimes shows that the skin layer recovery is a function of the initial condition of the skin layer and the net heat flux as well as of the strength of the disturbance. The initial condition of the skin layer is determined largely by the background shear-induced turbulence in the upper ocean. We have shown that for environmental conditions that remained constant, the restoring internal energy flux varied less than an order of magnitude as a function of the strength of the disruption. However, as environmental conditions such as wind stress and net heat flux became more intense, the mean $J_{r}$ increased roughly an order of magnitude. The enhanced background turbulence accelerated the net heat flux and transferred more heat from the surface and out of the recovering skin layer. Furthermore, the turbulence generated by the disruption in excess of the background turbulence was considerably less than that generated under low or no wind conditions. Since more background turbulence existed, the excess turbulence diffused more rapidly, increasing the restoring internal energy flux. In fact, although $Q_{\text {net }}$ increased only $36 \%$ from the ordered wake case to the wispy wake and breaking-wave cases, the average $J_{r}$ increased by a factor of 20 , implying that the increased recovery rate was due mostly to background turbulence rather than to $Q_{\text {ner }}$ This implies that for the same $Q_{\text {net }}$ and strength of the disturbance, $J_{r}$ will be greater when the background turbulence is greater.

\section{Conclusions}

Measurements of skin temperature made aboard R/P Flip show that infrared imagery is a useful tool for visualizing and quantifying turbulent disruptions of an air-water interface. We measured the infrared signatures of wakes from cables and found that the timescale for the thermal recovery of the skin layer is related to the strength of the wave-induced disruption in terms of a wave Reynolds number $R e_{w}$. The size and shape of the disturbances produced by the cables were strongly dependent on the oscillatory characteristics of the flow due to the presence of waves and surface currents. As $R e_{w}$ increased, the patches became larger, persisted longer, and reached their peak size later. An artificial disturbance of the skin layer by a cable of $O\left(10^{-2} \mathrm{~m}\right)$ in diameter produced turbulent patches of $O(1 \mathrm{~m})$ in diameter. These patches persisted for up to $80 \mathrm{~s}$ before drifting out of the field of view of the imager without fully recovering and were estimated to have lifetimes of up to $120 \mathrm{~s}$. The measurements substantiated the existence of the skin layer up to wind speeds of $10 \mathrm{~m} \mathrm{~s}^{-1}$.

We have demonstrated the distinct difference between the skin layer recovery time $t_{*}$ and the surface-renewal timescale $\tau$ (see Figure $1 \mathrm{~b}$ ). Whereas $t_{*}$ represents a response to a disruptive event and the decay of turbulence, $\tau$ reflects the timescale for physical replacement of surface water by turbulent eddies.
Nonetheless, $t_{*}$ values measured at low wind speed for ordered free-surface wakes were comparable to the parameterized timescale for surface renewal based on free convection $\tau_{c}$. However, at moderate wind speeds the difference between $t_{*}$ and $\tau$ becomes apparent. For both wispy, free-surface wakes and breaking waves, $t_{*}$ is an order of magnitude less than the parameterized shear-induced timescale $\tau_{s}$. Other than the explicit physical difference between $t_{*}$ and $\tau$, a possible reason for this discrepancy is that $\tau_{s}$ is not an appropriate parameterization for $\tau$. Therefore, if $t_{*}$ is to be used as an estimate for $\tau$ at moderate wind speeds, mechanisms responsible for the enhancement of surface renewal such as microscale and large-scale wave breaking need to be addressed in future parameterizations of $\tau_{s}$. Recent measurements by Jessup et al. [1997b] suggest that the strong eddies that have been associated with surface renewal in wind wave experiments [Komori et al., 1993] may be due to microscale wave breaking.

The restoring internal energy flux $J_{r}$, which is proportional to both the initial thickness and the thermal recovery rate of the skin layer, describes the rate of decrease in internal energy of a cool skin layer per unit area during the wake recovery. The restoring internal energy flux was found to be directly related to the strength of the disruption, represented in terms of a wave Reynolds number $R e_{w}$ for the ordered wakes. Laboratory measurements of breaking waves without wind showed comparable values for $J_{r}$ as a function of the energy dissipation rate $E$ at a constant air-water temperature difference $\Delta T_{a w}$. In the laboratory the restoring internal energy flux was correlated with the net heat flux since $J_{r}$ increased as a function of $\Delta T_{a w}$ for all $E$. Analysis of field measurements of wispy wakes and breaking waves at moderate wind speeds showed considerably greater restoring internal energy fluxes than the low wind speed results. The large variation of $J_{r}$ among these field and laboratory results suggests that the skin layer recovery rate is a strong function of the initial state of the skin layer, which relates directly to the existing environmental conditions (i.e., near-surface background turbulence and net heat flux). For a given set of environmental conditions the restoring internal energy flux varied less than an order of magnitude as a function of the strength of the disruption. For varying environmental conditions, however, $J_{r}$ potentially may vary by several orders of magnitude. Therefore the existing background turbulence, coupled with the net heat flux, plays a significant role in regulating the recovery rate of the skin layer and may prove to be more important than the strength of the disturbance.

\section{Appendix A: Reflection Effects}

In Figure 8 the infrared boom reflection causes an apparent change in sea surface temperature roughly $0.4^{\circ} \mathrm{C}$ greater than the background temperature, owing to the nonunity emissivity of the ocean surface. We found that, in general, the infrared reflection of the instrument boom corresponded directly to its visual reflection. While the boom's infrared reflection caused an apparent change in temperature, the instrument cables shown at top left of Figure 8 produced a real change by disrupting the cool skin layer. Under these low wind speed conditions the temperature changes within the wakes emanating from the cables and those induced by the boom reflection were comparable.

Simpson and Paulson [1980] made infrared measurements from R/P Flip by mounting a Barnes model PRT-5 radiometer with a narrow field of view at normal incidence on the boom. They argued that the effect of reflected radiation from the boom was negligible and was described by 


$$
\sigma T_{b r}^{4}=\sigma T_{s}^{4}+\Gamma \sigma T_{r}^{4}\left(\frac{r_{r}^{2}}{h^{2}}\right) \pi r_{s}^{2},
$$

where $T_{b r}$ is the brightness temperature measured by the radiometer, $T_{r}$ is the temperature of the boom, $\Gamma$ is the reflectivity of the ocean surface, $r_{r}$ is the radius of the spot on the boom's surface emitting radiation that is reflected into the radiometer, $h$ is the height of the boom, and $r_{s}$ is the radius of the radiometer spot at the ocean surface. In this discussion, (Al) is multiplied by the Stefan-Boltzmann constant $\sigma$ for clarity. Our observations have shown that boom reflection can be significant, and upon further analysis of (A1) we are able to provide an explanation for the disagreement with the conclusion of Simpson and Paulson [1980]. First, although the PRT-5 they used responded to radiation with wavelengths in the 8- to $14-\mu \mathrm{m}$ range, (A1) was derived from the Stefan-Boltzmann law which applies to emission over all wavelengths. Second, (A1) is dimensionally incorrect, since the term on the left-hand side and the first term on the right-hand side are derived from flux densities (watts per square meter) whereas the second term on the right-hand side is derived from radiant flux (watts). The radiant flux term is the source of underestimating the effect of boom reflection. Finally, the first term on the right-hand side of (A1) should be weighted by the emissivity of the ocean surface $\varepsilon_{S}$, which is a function of both wavelength and incidence angle. An average $\varepsilon_{s}$ value for $8-14 \mu \mathrm{m}$ and nadir incidence is roughly 0.985 .

$$
\begin{aligned}
\int_{8}^{14} M_{b r}\left(\lambda, T_{b r}\right) \partial \lambda= & \int_{8}^{14} \varepsilon_{s}(\lambda) M_{s}\left(\lambda, T_{s}\right) \partial \lambda \\
& +\int_{8}^{14} \varepsilon_{r}\left[1-\varepsilon_{s}(\lambda)\right] M_{r}\left(\lambda, T_{r}\right) \partial \lambda,
\end{aligned}
$$

In order to explain our boom reflection observations, we introduce the radiative balance used to determine $T_{s}$,

where $M$ is the Planck function for a Lambertian surface; $\lambda$ is wavelength; and the subscripts $b r, s$, and $r$ represent the brightness, surface, and reflected quantities, respectively. The brightness temperature in (A2) is composed of a portion emitted from the ocean surface and a portion reflected from either the sky, clouds, or the boom. Since the emissivity of the ocean surface is nearly constant at incidence angles less than $40^{\circ}$, we assume that changes in surface slope due to waves do not affect our results. For the ordered wakes shown in Figure 8a, $T_{r}$ is the sky temperature of $-18^{\circ} \mathrm{C}$ and $T_{s}$ is $13.7^{\circ} \mathrm{C}$, resulting in a $T_{b r}$ of $13.2^{\circ} \mathrm{C}$, assuming the sky behaves as a blackbody $\left(\varepsilon_{r}=1\right)$. For the boom reflection, $T_{r}$ is the temperature of the boom, which we estimate as $14.6^{\circ} \mathrm{C}$, since the air and water temperatures were the same and the $\varepsilon_{r}$ for the painted boom is 0.9 , resulting in a $T_{b r}$ of $13.6^{\circ} \mathrm{C}$. The brightness temperatures accounting for boom and sky reflection differ by $0.4^{\circ} \mathrm{C}$, which is identical to the difference observed in Figure 8a. Figure 8b shows similar deviations.

Values of $T_{s}$ that were corrected for sky reflection were calculated using (A2) [Zappa, 1994]. Early during the experiment aboard R/P Flip, a PRT-5 radiometer and a Heimann model KT19 radiometer mounted on the boom at a height of $10 \mathrm{~m}$ were aimed at an incidence angle of $5^{\circ}$ to measure the average $T_{b r}$ from a spot on the ocean that was $0.4 \mathrm{~m}$ in diameter. A video camera aligned with and in close proximity to the radiometers showed occasional visual reflection of the boom at low wind speeds. To avoid contamination by reflections from the instrument loom, the radiometers were switched to an incidence angle of $15^{\circ}$. An upward looking KT-19 aimed at the corresponding ze- nith angle was used to correct for sky reflection. Typically, a clear sky is much cooler than an overcast or cloudy sky. Consequently, the reflection corrections for these experimental data were as much as $0.5^{\circ} \mathrm{C}$ for clear skies. On the other hand, no correction was needed during overcast conditions since the warm clouds caused $T_{r}$ to be approximately equal to $T_{s}$, effectively compensating for the imperfect emission of the ocean surface.

The increase in surface roughness (i.e., gravity-capillary waves) generated by an increase in wind speed has the effect of smoothing out the abrupt temperature gradients due to reflection at the ocean surface. Saunders [1967b, Figure 4a] showed that the apparent increase arising from the reflection of direct solar radiance from the sea surface decreases with increasing surface roughness. Small-scale roughness increases with wind speed and creates a diffuse rather than a specular surface, which is prevalent under low winds. Therefore, since the length scale of the roughness is less than the resolution of the imager, the boom is only a small portion of what the ocean surface reflects. Figures 7 and 8 contrast the infrared reflection effect observed at high and low wind speeds during clear skies. Under low winds and negligible surface roughness (Figure 8) the specular reflection of the boom is clearly visible. Under higher winds and surface roughness (Figure 7) the boom reflection is indiscernible.

\section{Appendix B: Comparison of $\Delta T$ and $\Delta T_{w}$}

The maximum temperature difference across the patch generated by the disruption of the cool skin provided a direct measurement of the bulk-skin temperature difference $\Delta T$. Previous methods of measuring $\Delta T$ by artificially disrupting the skin layer used narrow field of view $\left(\sim 2^{\circ}\right)$ radiometers that provided a point measurement of the surface temperature [Ewing and $M c$ Alister, 1960; Hill, 1972; Katsaros, 1977]. Here we utilize the ability of the infrared imager to measure $\Delta T$ directly by simultaneously observing the temperature in the patches and in undisturbed areas within a single image. For comparison, we calculated $\Delta T$ as traditionally done by combining the radiometer measurements of $T_{s}$ and the thermistor measurements of $T_{b}$ at $0.1 \mathrm{~m}$ depth as outlined in section 2 and Appendix A. For the ordered wakes the maximum $\Delta T_{w}$ extracted from the images was $0.45^{\circ} \pm 0.05^{\circ} \mathrm{C}$ whereas the $\Delta T$ calculated using the radiometer and thermistor measurements was $0.55^{\circ} \pm 0.05^{\circ} \mathrm{C}$. This difference of $\pm 0.1^{\circ} \mathrm{C}$ is within the experimental error for the measurement of $T_{s}$ using the radiometers. In addition, since the thermistor was at a depth of $0.1 \mathrm{~m}$, well beyond the bottom of the skin layer, $\Delta T$ may have been overestimated. Since the maximum $\Delta T_{w}$ increased with $R e_{w}$, indicating that the skin layer may not have been completely destroyed at low $R e_{w}$, it is also possible that $\Delta T_{w}$ was underestimated. These factors provide additional confidence in the accuracy of the direct measurement of $\Delta T$ using infrared imagery via surface disruption. The wispy wakes provided a measure of $\Delta T$ at higher wind speeds. We estimated a maximum $\Delta T_{w}$ of $0.1^{\circ} \mathrm{C}$ from the infrared images, whereas the calculated $\Delta T$ was approximately $0.2^{\circ} \mathrm{C}$. Although the comparison is within experimental error, the skin layer recovered so quickly that the patches may not provide an accurate measure of $\Delta T$ at these higher wind speeds. When solar radiation is negligible, our direct technique may provide an alternative measurement of $\Delta T$ that does not require the measurement of the bulk temperature $T_{b}$ or the in-field calibration of radiometers [Schliussel et al., 1990; Suarez et al., 1997]. 
Acknowledgments. We thank our colleagues from the Applied Physics Laboratory (APL-UW) and the Department of Atmospheric Sciences, University of Washington, as well as the crew of R/P Flip for the assistance received during the field operation. We appreciate the insightful comments of G. A. Wick and W. E. Asher. This work was funded by grants from the Office of Naval Research, the National Aeronautics and Space Administration, and APL-UW.

\section{References}

Csanady, G. T., The role of breaking wavelets in air-sea gas transfer, $J$. Geophys. Res., 95, 749-759, 1990.

Danckwerts, P. V., Significance of liquid-film coefficients in gas absorption, Ind. Eng. Chem., 43, 1460-1467, 1951.

Ewing, G., and E. D. McAlister, On the thermal boundary layer of the ocean, Science, 131, 1374-1376, 1960.

Fairall, C. W., E. F. Bradley, D. P. Rogers, J. B. Edson, and G. S. Young, Bulk parameterization of air-sea fluxes for Tropical Ocean Global Atmosphere-Coupled Ocean Atmosphere Response Experiment, $J$. Geophys. Res., 101, 3747-3764, 1996.

Foster, T. D., Intermittent convection, Geophys. Fluzd Dyn., 2, 201-217, 1971.

Gulliver, J. S., Introduction to air-water mass transfer, in Air-Water Mass Transfer, edited by S. C. Wilhelms and J. S. Gulliver, pp. 1-7, Am. Soc. Civ. Eng., New York, 1991.

Harriott, P., A random eddy modification of the penetration theory, Chem. Eng. Sci., 17, 149-154, 1962.

Harris, A. R., and M. A. Saunders, Global validation of the along-track scanning radiometer against drifting buoys, J. Geophys. Res., 101, $12,127-12,140,1996$.

Hasse, L., On the mechanism of gas exchange at the air-sea interface, Tellus, 42B, 250-253, 1990.

Hill, R. H., Laboratory measurement of heat transfer and thermal structure near an air-water interface, J. Phys. Oceanogr., 2, 190-198, 1972.

Jahne, B., P. Libner, R. Fischer, T. Billen, and E. J. Plate, Investigating the transfer processes across the free aqueous viscous boundary layer by the controlled flux method, Tellus, Ser. B, 41B, 177-195, 1989.

Jessup, A. T., C. J. Zappa, V. Hesany, M. R. Loewen, and M. G. Skafel, Dependence of the skin layer recovery rate on heat flux and turbulence, Air-Water Gas Transfer, edited by B. Jahne and E. C. Monahan, pp. 601-610, Aeon, Hanau, Germany, 1995.

Jessup, A. T., C. J. Zappa, M. R. Loewen, and V. Hesany, Infrared remote sensing of breaking waves, Nature, 385, 52-55, 1997a.

Jessup, A. T., C. J. Zappa, and H. Yeh, Defining and quantifying microscale wave breaking with infrared imagery, J. Geophys. Res., 102, 23,145-23, 154, 1997b.

Katsaros, K. B., The sea surface temperature deviation at very low wind speeds; Is there a limit?, Tellus, 29, 229-239, 1977.

Katsaros, K. B., The aqueous thermal boundary layer, Boundary Layer Meteorol., 18, 107-127, 1980.

Katsaros, K. B., W. T. Liu, J. A. Businger, and J. E. Tillman, Heat transport and thermal structure in the interfacial boundary layer measured in an open tank of water in turbulent free convection, J. Fludd Mech., $83,311-335,1977$

Keller, W. C., and W. J. Plant, Cross sections and modulation transfer functions at $L$ and $K_{u}$ bands measured during the Tower Ocean Wave and Radar Dependence Experiment, $J$. Geophys. Res., 95, 16,277$16,289,1990$

Komori, S., R. Nagaosa, and Y. Murakami, Turbulence structure and mass transfer across a sheared air-water interface in wind-driven turbulence, J. Fluid Mech., 249, 161-183, 1993.

Konda, M., N. Imasato, K. Nishi, and T. Toda, Measurement of the sea surface emissivity, J. Oceanogr., 50, 17-30, 1994.

Kudryavtsev, V. N., and A. V. Soloviev, On the parameterization of the cold film on the ocean surface, Izv. Acad. Sci. USSR Atmos. Oceanic Phys., Engl. Transl., 21, 177-183, 1985.

Liu, W. T., K. B. Katsaros, and J. A. Businger, Bulk parameterization of air-sea exchanges of heat and water vapor including the molecular constraints at the interface, J. Atmos. Sci., 36, 1722-1735, 1979.

McAlister, E. D., and W. McLeish, Heat transfer in the top millimeter of the ocean, J. Geophys. Res., 74, 3408-3414, 1969.

Robertson, J. E., and A. J. Watson, Thermal skin effect of the surface ocean and its implications for $\mathrm{CO}_{2}$ uptake, Nature, 358, 738-740, 1992.

Robinson, I. S., N. C. Wells, and H. Charnock, The sea surface thermal boundary layer and its relevance to the measurement of sea surface temperature by airborne and spacebome radiometers, Int. J. Remote Sens., 5, 19-45, 1984.

Saunders, P. M., Aerial measurement of sea surface temperature in the infrared, $J$. Geophys. Res., 72, 4109-4117, 1967a.

Saunders, P. M., The temperature at the ocean-air interface, J. Atmos. Sci., 24, 269-273, $1967 \mathrm{~b}$.

Schlüssel, P., W. J. Emery, H. Grassl, and T. Mammen, On the bulk-skin temperature difference and its impact on satellite remote sensing of sea surface temperature, J. Geophys. Res., 95, 13,341-13,356, 1990.

Simpson, J. J., and C. A. Paulson, Small-scale sea surface temperature structure, J. Phys. Oceanogr., 10, 399-410, 1980.

Soloviev, A. V., Coherent structures at the ocean surface in convectively unstable conditions, Nature, 346, 157-160, 1990.

Soloviev, A. V., and P. Schlussel, Parameterization of the cool skin of the ocean and of the air-ocean gas transfer on the basis of modeling surface renewal, J. Phys. Oceanogr., 24, 1339-1346, 1994.

Suarez, M. J., W. J. Emery, and G. A. Wick, The multi-channel infrared sea truth radiometric calibrator (MISTRC), J. Atmos. Oceanic Technol., 14, 243-253, 1997.

Van Scoy, K. A., K. P. Morris, J. E. Robertson, and A. J. Watson, Thermal skin effect and the air-sea flux of carbon dioxide: A seasonal high-resolution estimate, Global Biogeochem. Cycles, 9, 253-262, 1995.

Wick, G. A., and A. T. Jessup, Simulation of ocean skin temperature modulation by swell waves, J. Geophys. Res., 103, 3149-3161, 1998.

Wick, G. A., W. J. Emery, L. H. Kantha, and P. Schlussel, The behavior of the bulk-skin sea surface temperature difference under varying wind speed and heat flux, J. Phys. Oceanogr., 26, 1969-1988, 1996.

$\mathrm{Wu}, \mathrm{J}$., An estimation of oceanic thermal-sublayer thickness, $J$. Phys. Oceanogr., 1, 284-286, 1971.

Zappa, C. J., Infrared field measurements of sea surface temperature: Analysis of wake signatures and comparison of skin layer models, M.S. thesis, Univ. of Wash., Seattle, 1994.

A. T. Jessup and C. J. Zappa, Applied Physics Laboratory, University of Washington, 1013 N.E. 40th Street, Seattle, WA 98105-6698. (e-mail: jessup@apl.washington.edu; zappa@apl.washington.edu)

H. Yeh, Department of Civil Engineering, University of Washington, Seattle, WA 98195-2700. (e-mail: harryeh@u.washington.edu)

(Received November 17, 1997; revised May 11, 1998 ; accepted June 5, 1998.) 